\title{
A set of common movements within GPCR-G-protein complexes from variability analysis of cryo-EM datasets
}

\author{
Jacopo Marino ${ }^{a, *}$, Gebhard F.X. Schertler ${ }^{a, b}$ \\ ${ }^{a}$ Laboratory of Biomolecular Research, Paul Scherrer Institut, Förschunggstrasse 111, 5232 Villigen, Switzerland \\ ${ }^{\mathrm{b}}$ Department of Biology, ETH Zürich, Wolfgang-Pauli-Strasse 27, Zürich 8093, Switzerland
}

\section{A R T I C L E I N F O}

\section{Keywords:}

GPCR

G-protein

Cryo-EM

Variability analysis

Inherent flexibility

Membrane proteins

\begin{abstract}
A B S T R A C T
G-protein coupled receptors (GPCRs) are among the most versatile signal transducers in the cell. Once activated, GPCRs sample a large conformational space and couple to G-proteins to initiate distinct signaling pathways. The dynamical behavior of GPCR-G-protein complexes is difficult characterize structurally, and it might hinder obtaining routine high-resolution density maps in single-particle reconstructions. Here, we used variability analysis on the rhodopsin- $\mathrm{G}_{\mathrm{i}}$-Fab16 complex cryo-EM dataset, and the results provide insights into the dynamic nature of the receptor-complex interaction. We compare the outcome of this analysis with recent results obtained on the cannabinoid- $\mathrm{G}_{\mathrm{i}^{-}}$and secretin- $\mathrm{G}_{\mathrm{s}}$-receptor complexes. Despite differences related to the biochemical compositions of the three samples, a set of consensus movements emerges. We anticipate that systematic variability analysis on GPCR-G-protein complexes may provide useful information not only at the biological level, but also for improving the preparation of more stable samples for cryo-EM single-particle analysis.
\end{abstract}

\section{Introduction}

G protein-coupled receptors (GPCRs) constitute the most versatile and diverse membrane protein family, with around 800 genes in humans. GPCRs recognize a large variety of extracellular stimuli, and once activated, initiate intracellular signaling cascades to trigger pivotal cellular responses (Sexton, 2018). Those responses include vision, smell, taste, secretion, nerve system regulation, cellular differentiation, and immune response. As consequence, diseases caused by malfunctioning of GPCRs are very diverse and have dramatic outcomes, including cancer. While $~ 30 \%$ of FDA-approved medications target GPCRs, an increasing number of lead compounds are on clinical trials (Hauser et al., 2017). However, pharmacological intervention is greatly limited by our understanding of the GPCR signaling pathway, which is complex and only partially understood (Capper and Wacker, 2018; Wacker et al., 2017). There is strong interest in rationalizing the GPCR signally pathway, as this information will undoubtedly translate into better understanding of biased agonism (Rajagopal et al., 2010; Violin et al., 2014) and ultimately to the design of therapeutics with better specificity and less side-effects (Hauser et al., 2017). Within a simplified picture, GPCR signaling is propagated trough trimeric $\mathrm{G} \alpha \beta \gamma$-proteins, where $\mathrm{G} \alpha$ belongs to four major families, $\mathrm{G}_{\mathrm{i} / \mathrm{o}}, \mathrm{G}_{\mathrm{s}}, \mathrm{G}_{\mathrm{q} / 11}$ and $\mathrm{G}_{12 / 13}$ (Costa-Neto et al., 2016). Activated GPCRs associate to trimeric G proteins, inducing exchange of GDP to GTP, and dissociation of G $\alpha$ from G $\beta \gamma$, triggering intracellular cascades (Wacker et al., 2017). Activated GPCRs are subsequently phosphorylated mostly at their C-terminus by G-protein receptor kinases (Gurevich et al., 2012), and the arrestin signaling pathway follows (Azevedo et al., 2015; Gurevich and Gurevich, 2019; Gurevich et al., 2012). The unlocking of the molecular basis for G-protein selectivity has been initially approached by sequence analysis (Flock et al., 2017). Developments in the biochemistry of GPCRs-Gprotein complexes, coupled to the selection of stabilizing binders $(\mathrm{Qu}$ et al., 2019; Thal et al., 2018), and advances in the field of cryo-electron microscopy (Danev et al., 2020) have resulted in the deposition of more than 300 entries in the Protein Data Bank (PDB), including structures of receptors coupled to long-awaited signaling partners (Huang et al., 2020; Lee et al., 2020; Maeda et al., 2019; Nguyen et al., 2019), and a variety of orthosteric and allosteric ligands (Qu et al., 2019). This growing information allows to draw stronger conclusions obtained from structural alignments of GPCRs bound to different classes of G-proteins (García-Nafría and Tate, 2019; Glukhova et al., 2018; Tsai et al., 2019). These analyses reveal the existence of conserved amino-acid contacts between TM3, TM5, and TM6 of GPCRs, and the C-terminal $\alpha 5$ helix of $\mathrm{G} \alpha$ proteins, with some contacts being G-protein-subtype specific.

\footnotetext{
* Corresponding author.

E-mail address: jacopo.marino@psi.ch (J. Marino).
} 
However, the interaction between GPCR and G-protein selectivity goes beyond a simple key-and-lock mechanism mediated by the $\alpha 5 \mathrm{C}$-terminal helix, and other conserved contacts between GPCRs and G-protein, especially those mediated by intracellular loop 2 (ICL2) and intracellular loop 3 (ICL3) might be important to ensure proper complex formation (Glukhova et al., 2018; Klenk et al., 2020; Tsai et al., 2019).

The versatility of GPCRs in responding to different ligands, and their capability of coupling to different intracellular partners lies within their dynamic nature (Deupi and Kobilka, 2010; Latorraca et al., 2017). Studying the structural dynamics of signaling complexes is a difficult task, and many activated states are transient (Gusach et al., 2020).

Obtaining atomic structures of GPCRs, and at the same time retain information about their conformational dynamics, is a difficult task. Xray free electron lasers (XFELs) have been used to study dynamics of GPCRs (Nass Kovacs et al., 2019; Nogly et al., 2018) as the methodology allows to record movies at very small time scales. However, molecules embedded in the crystal lattice can explore a limited conformational space. Obtaining crystals for GPCR-G-protein complexes is also difficult especially due to the conformational heterogeneity naturally present in the sample which will hinder crystal formation.

Within the field of cryogenic electron microscopy (cryo-EM), timeresolved cryo-EM has been employed to resolve conformational states after protein activation (Kontziampasis et al., 2019; Unwin and Fujiyoshi, 2012). Cryo-EM is suitable for studying conformational heterogeneity (Lyumkis, 2019) because the landscape of conformational states is preserved during sample vitrification (Glaeser, 2018). In standard single-particle analysis, thousands of 2D particles images are used to refine a single, consensus density map to high resolution. However, under these conditions, information about flexibility and conformational heterogeneity is restricted to 3D-classification, within the limitations of the method (Haie-Meder, 1998; Scheres, 2016). Resolving intrinsic structural heterogeneity from 2D particle images is computationally difficult, and several approaches have been proposed over the last few years (Andén and Singer, 2018; Dashti et al., 2014; Jin et al., 2014; Penczek et al., 2011; Tagare et al., 2015; Zhang et al., 2019). In a simplified picture, structural heterogeneity within a cryo-EM sample lies within the manifold of its energetically permitted 3D structures, which can be simplified into a linear K-dimensional subspace model. In this model, each particle image is assumed to be composed by its mean density $\mathrm{V}_{\mathrm{o}}$ plus a contribution from the variability components $\mathrm{V}_{1: \mathrm{K}}$. $3 \mathrm{D}$ variability analysis, as deeper described in the preprint from Punjani and Fleet (Punjani and Fleet, 2020), is based on probabilistic principal component analysis (PPCA), where data is described by a Gaussian distribution. When this model is applied to single-particle EM-data, the output will contain the components $\mathrm{V}_{1: \mathrm{K}}$ and the reaction coordinates $\mathrm{z}$. The variability components are reflected into density values (positive or negative relative to $V_{0}$ ) in each voxel of the density maps, and can be used to visualize to which extent variability takes place within the original 2D particle images. The algorithm allows to explore, within a cryo-EM dataset, particle flexibility and heterogeneity while retaining high-resolution information, including more difficult samples such as small membrane proteins, which is particularly relevant to study structural flexibility of GPCR-G-protein complexes.

At the time of writing of this article, 3D-variability analysis has been reported for the cannabinoid-1 (Punjani and Fleet, 2020), the secretin (Dong et al., 2020), and the adrenomedullin (Liang et al., 2020) receptor-G-protein complexes. Here, we compare the outcome of these analysis, and report on the results obtained by performing the same analysis on the rhodopsin- $\mathrm{G}_{i} \alpha \beta \gamma$-Fab16 complex dataset (Tsai et al., 2019). Interestingly, a set of common movements is shared among the GPCR complexes taken into consideration.

\section{Materials and methods}

A particle stack of 111,357 images from the consensus refinement performed in Relion (Tsai et al., 2019) was imported into cryoSPARC
(Punjani et al., 2017) using pyem (Asarnow et al., 2019). An $a b$-initio map was produced, which was used as input for refinement using a mask that includes flexible regions (AH-domain of $G_{i} \alpha$, and part of $G_{i} \alpha$ below TM5-TM6 of rhodopsin) that were previously excluded to achieve higher resolution (Tsai et al., 2019). For 3D-variability analysis, a mask that excludes the detergent micelle was created (Supplementary Fig. 1A), as previously described (Punjani and Fleet, 2020). For consistency with the other two published analysis with which comparisons are made (Dong et al., 2020; Punjani and Fleet, 2020), three modes were indicated, and the output contained for each mode 20 density maps, which were used for creating movies in Chimera (Pettersen et al., 2004). Results were filtered at 6 Angstrom. The first and last frames (frame 0 and frame 19), corresponding to negative and positive values of the reaction coordinate for each variability component (Punjani and Fleet, 2020) were used for displaying differences within each component.

\section{Results}

\subsection{D-variability analysis of the rhodopsin- $G_{i}$-Fab16 complex}

The results of the 3D-variability analysis obtained on the rhodopsin$\mathrm{G}_{\mathrm{i}} \alpha \beta \gamma$-Fab16 dataset are now described. The first component resolves a large movement of the $\mathrm{G}_{\mathrm{i}} \alpha$ alpha-helical (AH) domain, a folding unit characteristic of G $\alpha$ proteins (Neol et al., 1993), which due to its flexible connection to the Ras domain remains invisible in most structures. As shown in Fig. 1, within the 3D reconstructions of the variability component one, the AH-domain is strongly visible in frame zero (blue) where it associates to $\mathrm{G} \beta$, but then becomes invisible in frame 19 (yellow), where it moves away from G $\beta$ (Fig. 1A, and Suppl. Video 1), thus resembling fluctuations between an open- and closed-conformation observed previously (Dror et al., 2015; Sun et al., 2018). We previously observed the presence of the AH-domain within the rhodopsin-Gprotein complex during 3D classification in Relion in one of the four 3Dclasses obtained (Tsai et al., 2019). Variability analysis reveals that the movement of the AH-domain is associated to twisting of rhodopsin around the axis parallel to the membrane, with larger movements especially visible for transmembrane (TM) segment 4 and TM6 (Fig. 1B and C, Suppl. Movie 1).

The second component of the variability analysis resolves bending of rhodopsin $\sim 20^{\circ}$ away from $\mathrm{G} \beta$ along the axis perpendicular to the membrane, and Fab16 and rhodopsin oscillates against each other (Fig. 2A). Interestingly, when rhodopsin bends towards $\mathrm{G} \beta \gamma$ (blue), intracellular loop 2 (ICL2) contacts the N-terminal helix of $G_{i} \alpha$, an interaction absent when rhodopsin is bent away (yellow) (Fig. 2B). The interaction of ICL2 with the N-terminal helix of $G_{i} \alpha$ is a recurrent feature observed in structures of GPCR-G-protein complexes, and it might be needed to stabilize the receptor-G-protein interface (García-Nafría and Tate, 2019; Glukhova et al., 2018; Tsai et al., 2019). Bending of rhodopsin away from G $\beta \gamma$ produces the effect of allowing TM5 and TM6 to get closer to $\mathrm{G}_{\mathrm{i}} \alpha$ (Fig. 2C), thus extending the interaction of ICL3 with $\mathrm{G}_{\mathrm{i}} \alpha$. The interaction of ICL3 with $\mathrm{G}_{\mathrm{i}} \alpha$ is also a feature that has been already observed in other GPCR-G-protein complexes (García-Nafría and Tate, 2019; Glukhova et al., 2018).

Interestingly, bending of the receptor away from Fab16 allows to visualize the density corresponding to the rhodopsin C-terminal tail (Ctail), which in our rhodopsin-complex (Tsai et al., 2019) and in the muscarinic receptor complex (Maeda et al., 2019) is bound to G $\beta$. Thus, our analysis shows that the interaction of the $\mathrm{C}$-tail to $\mathrm{G} \beta$ might be dynamic. The density corresponding to rhodopsin' C-tail is not visible when the receptor bends towards Fab16 (blue), also at very low thresholds (Fig. 2D and Suppl. Fig. 2), indicating that rhodopsin must be at a closer distance to $\mathrm{G} \alpha \beta$ to stabilize the C-tail in that position.

Another observation that can be made within these reconstructions is that in frame zero of component two, a density growing from $\mathrm{G} \beta$ to the receptor micelle is visible (Fig. 2D). This density may be attributed to posttranslational modifications of $\mathrm{G} \beta \gamma$ interacting with the detergent 


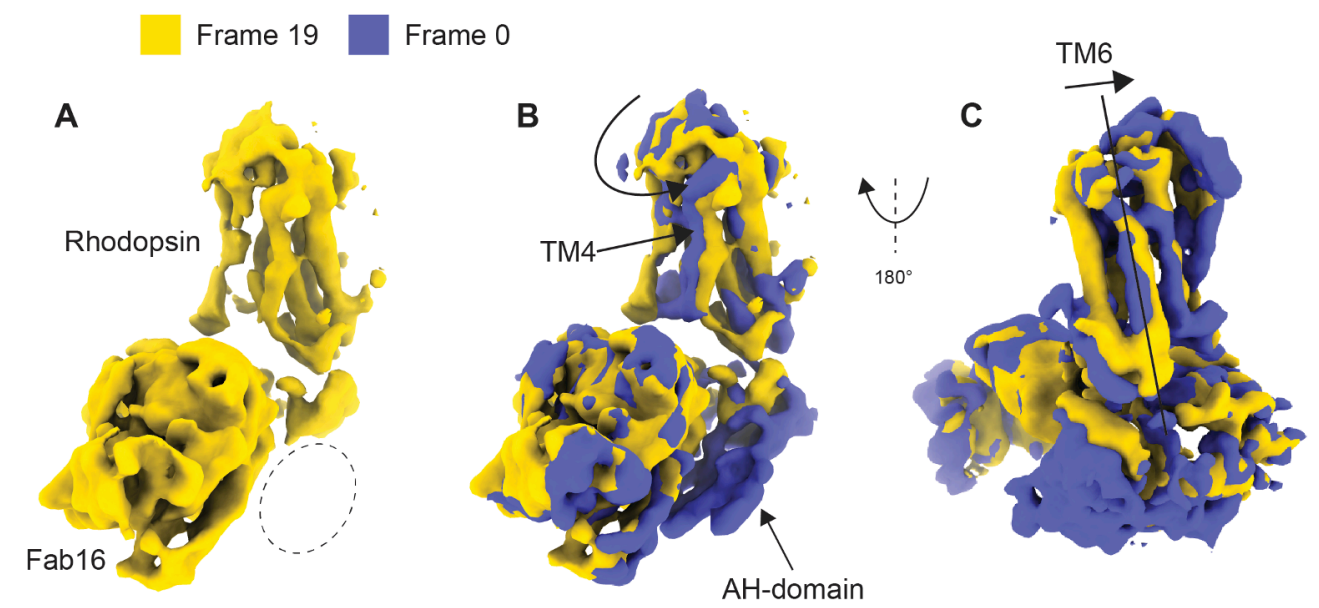

Fig. 1. Result component 1 of cryoSPARC 3D variability analysis of the rhodopsin- $\mathrm{G}_{\mathrm{i}}$-complex bound to Fab16. A) Density map corresponding to frame 19 of the output of component one. In circle is indicated the missing density of the alpha-helical (AH) domain, which is instead visible in frame zero (B). Component one also resolves rotation of rhodopsin along the membrane axis, with larger displacement of TM4 (B) and TM6 (C).
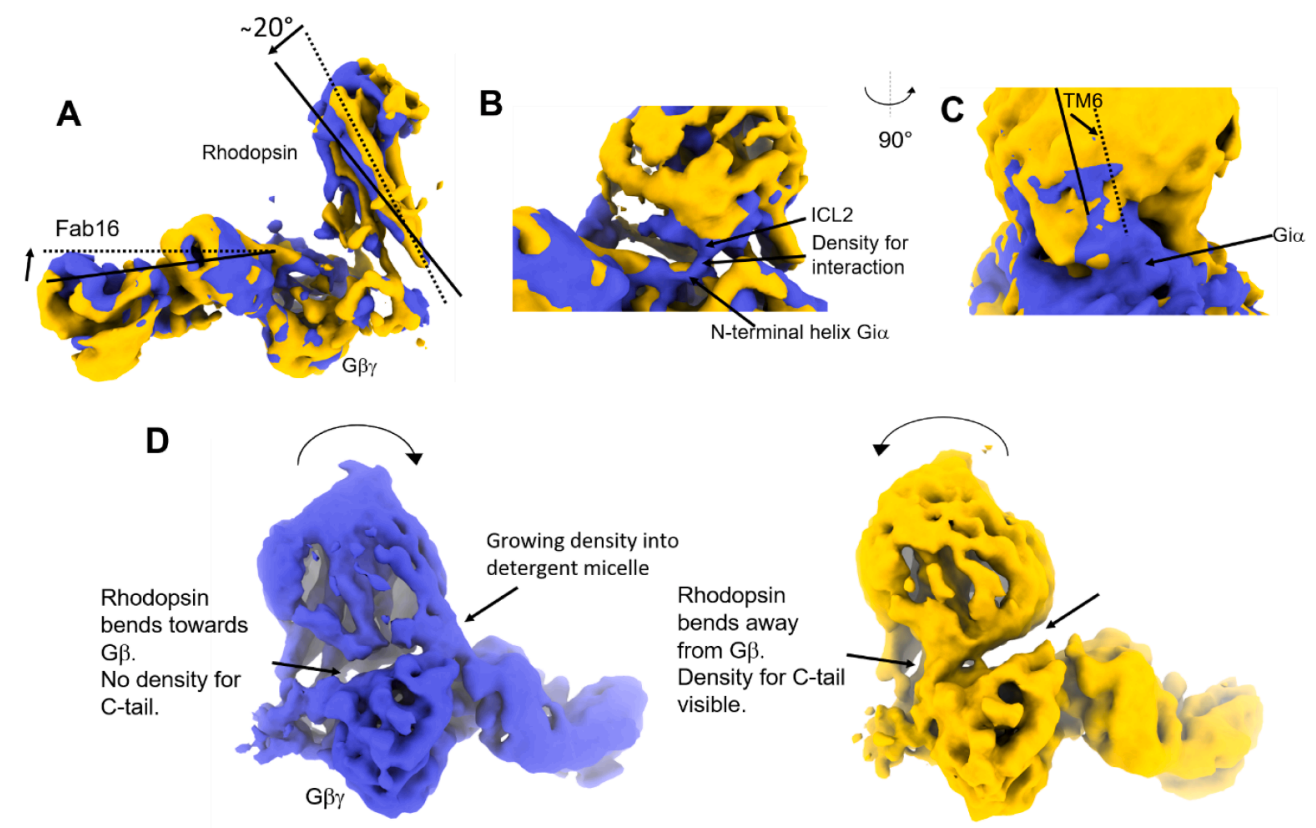

Fig. 2. Result component two. A) Representation of the two density maps corresponding to frame zero (blue) and frame 19 (yellow) resulting from component two. The oscillating movement of rhodopsin towards Fab16 is indicated. In B) is shown the density growing from the N-terminal helix of $\mathrm{G}_{\mathrm{i}} \alpha$ , which is not visible in frame 19 (yellow). In C), rotation of rhodopsin causes TM6/ICL3 to make a larger contact area with $\mathrm{G}_{\mathrm{i}} \alpha$. In D) is indicated the absence (blue) or presence (yellow) of density corresponding to the rhodopsin C-terminal tail (see also Suppl. Fig. 2), and presence (blue) or absence (yellow) of density which might be attributed to posttranslational modifications of $\mathrm{G} \beta \gamma$. micelle (Vögler et al., 2008), as G $\beta \gamma$ was purified from bovine retina and thus posttranslational modifications should be retained (Tsai et al., 2019). This density is not present when the receptor bends away from G $\beta \gamma$ (Fig. 2D, yellow), also at lower thresholds (Suppl. Movie 2).

Component three resolves transversal bending of rhodopsin, Fab16 and $\mathrm{G} \beta \gamma$. Differently from the movements observed in component two, rhodopsin does not bend towards $\mathrm{G} \beta \gamma$ and Fab16, but rather oscillates towards the N-terminal helix of $\mathrm{G}_{\mathrm{i}} \alpha$. Here, $\mathrm{G} \beta \gamma$ and Fab16 rotate of $\sim 15^{\circ}$ (Fig. 3A). The rotation of Fab16 propagates on its anchoring points, which are $\mathrm{G} \beta$ and the $\mathrm{N}$-terminal helix of $\mathrm{G}_{i} \alpha$. The $\mathrm{N}$-terminal helix of $\mathrm{G}_{i} \alpha$ thus translates vertically, as indicated in Fig. 3B, thus pulling rhodopsin up and down. The relative video is provided as Suppl. Movie 3.

\subsection{Comparison with reported 3D-variability analysis of GPCR complexes}

The movements observed within the rhodopsin- $\mathrm{G}_{\mathrm{i}}$-Fab16 complex can be summarized as it follows. The first component resolves twisting of rhodopsin along an axis parallel to the membrane, accompanied by a large movement of the AH-domain. The second component resolves bending of the receptor against Fab16, and the forming and breaking of interactions at the receptor-G-protein interface; the third and last component resolves a rotation of Fab16 and G $\beta \gamma$, which lead to transverse bending of rhodopsin (Fig. 4A).

Punjani and Fleet have applied 3D-variability analysis to the cannabinoid receptor $1-\mathrm{G}_{\mathrm{i}}$-protein complex dataset (Krishna Kumar et al., 2019) (EMPIAR: 10288). . In their study, the analysis in restricted to three components, and in Fig. 4B we report a schematic representation of the moments that the authors describe (Punjani and Fleet, 2020). The first component shows that the cannabinoid receptor bends towards G $\beta \gamma /$ Fv16; the second component resolves a motion of G $\beta \gamma$ with transversal bending of the receptor, while the third component resolves twisting of the receptor parallel to the membrane axis (Fig. 4B).

In a recent study, Dong and colleagues performed 3D variability analysis on the human secretin receptor coupled to $\mathrm{G}_{s}$ (Dong et al., 2020). Their analysis takes into consideration three components, and they show very similar movements as compared to the cannabinoid receptor complex. The first component resolves rotation of the receptor 

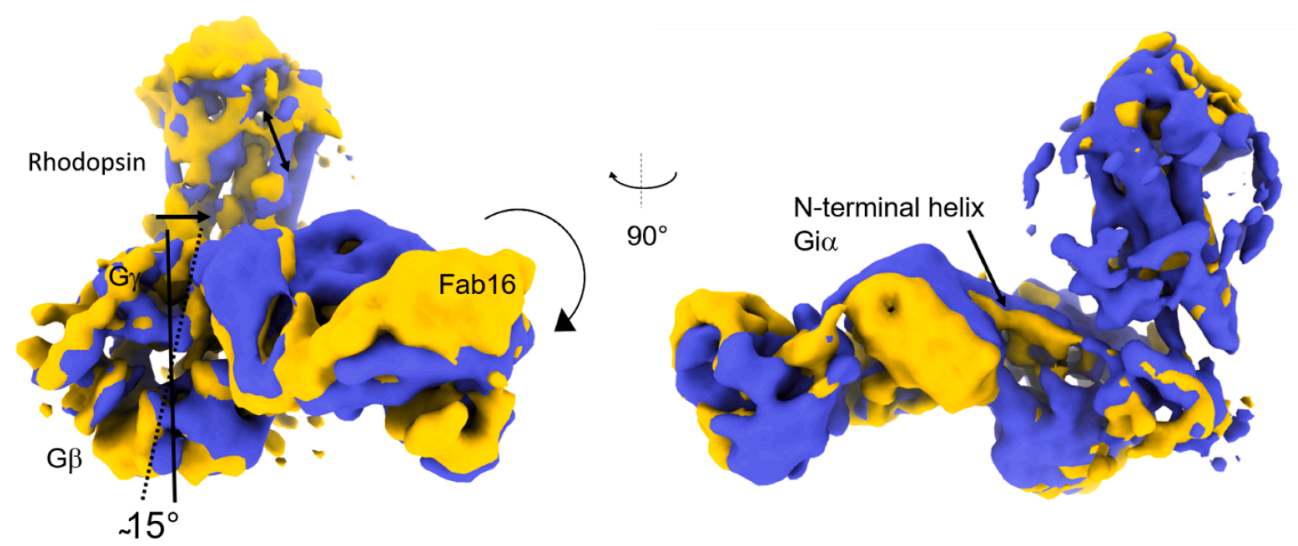

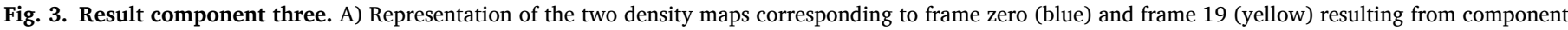

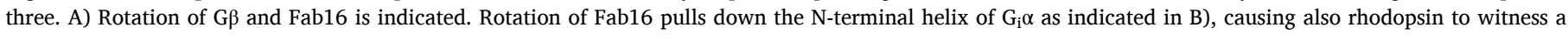
transversal movement of similar amplitude.

Rhodopsin-Gi-Fab16 complex

A
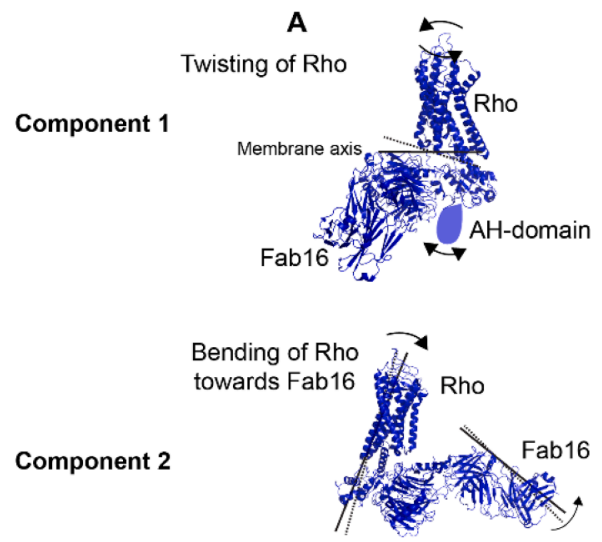

Transverse bending of Rho
and $G \beta \gamma-F a b 16$

Component 3

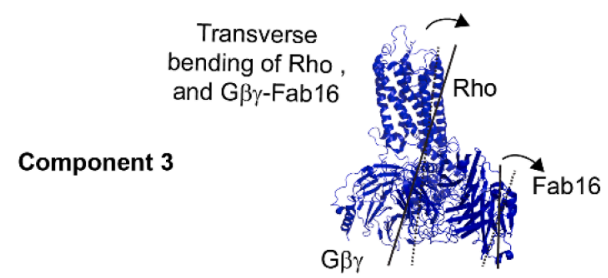

Cannabinoid-1-Gi-scFv16 complex

B
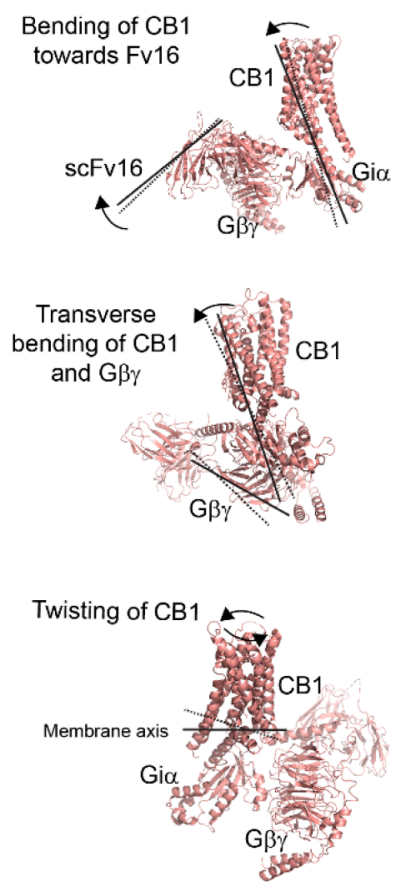

secretin-Gs-Nb35 complex

C
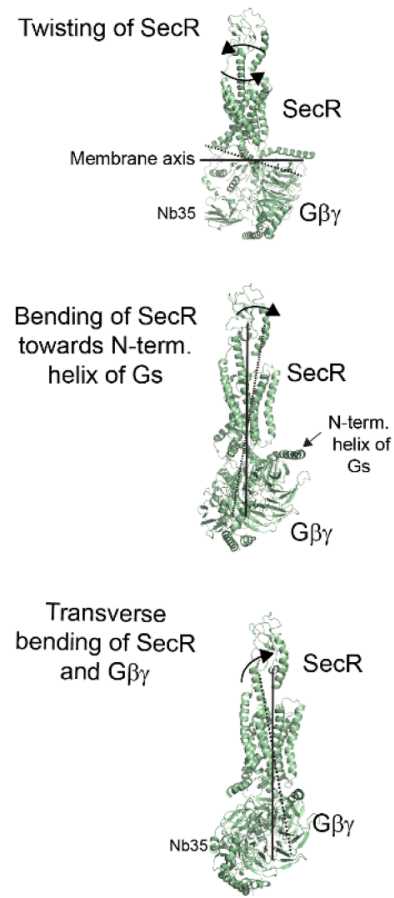

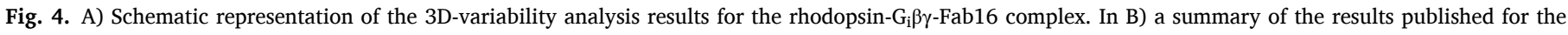

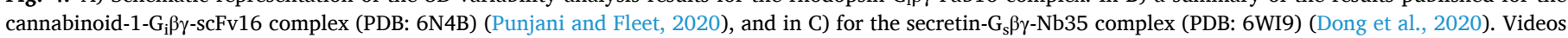

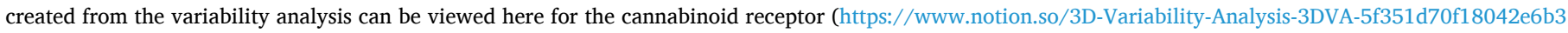

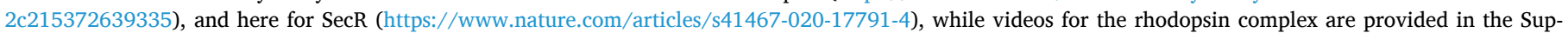
plementary Materials.

along the axis parallel to the membrane. The second component resolves bending of the receptor towards the N-terminal helix of $\mathrm{G}_{\mathrm{s}}$ (or towards nb35), a movement with similar amplitude to what observed in component 1 of the cannabinoid receptor analysis. We speculate that the different bending direction of the secretin receptor in comparison to rhodopsin and cannabinoid receptors -which instead bend towards Fab16 - might be due to the presence of the long and rigid secretin receptor' C-terminal helix which lies parallel to G $\beta$. Finally, the third component resolves transverse bending of the receptor (Fig. 4C). The bending movement of the secretin receptor towards the N-terminal helix of $\mathrm{G}_{\mathrm{s}}$ is nearly identical to what observed within the Adrenomedullin receptors $\left(\mathrm{AM}_{1}\right.$ and $\mathrm{AM}_{2}$ ) (Liang et al., 2020), as well a class B GPCR, and have nb35 bound to $G_{s}$. Thus, despite their biological and biochemical differences, the overall movements identified by 3D-variability analysis share similarities. 


\section{Discussion}

Proteins adopt different conformations along the energy landscape to perform their biological function. Protein flexibility, including disorder, is key to explain molecular machines at work. The complex signal transduction pathway across the cell membrane initiated by G-proteincoupled receptors (GPCRs) is a showcase for transient protein interactions, and structural pharmacology would benefit from characterizing the molecular basis for the transient interactions taking place after GPCR activation. This information will undoubtedly contribute to rationalizing G-protein selectivity and biased agonism (Sexton and Christopoulos, 2018), translating into the development of more specific treatments (Hauser et al., 2017).

Transient interactions between GPCR and G-proteins are difficult to capture by standard structural biology approaches. These events, among others, include G-protein pre-coupling and selectivity, and short-living GDP-bound states (Gusach et al., 2020). The study of dynamics of activated GPCRs has been approached with different techniques including NMR, EPR, FRET, and X-FEL (Gusach et al., 2020). Within true cellular membranes, valuable insights have been produced by single-molecule imaging of GPCR complexes (Sungkaworn et al., 2017).

Cryo-electron microscopy has streamlined many steps to solve atomic-resolution structures of GPCR-G-protein complexes (Danev et al., 2020), and an advantage about cryo-EM is that information about inherent flexibility and sample heterogeneity is preserved within cryoembedded particles. New algorithms to extract information about dynamics within cryo-EM datasets are providing useful insights about motions connected to biological function (Dashti et al., 2020; Frank and Ourmazd, 2016; Nešić et al., 2020; Ramírez et al., 2019; Rogala et al., 2019; Stanishneva-Konovalova et al., 2020; Wrapp et al., 2020), including for GPCR-G-protein complexes (Dong et al., 2020; Liang et al., 2020; Punjani and Fleet, 2020).

Here, we report on the outcome of variability analysis performed on the rhodopsin- $\mathrm{G}_{\mathrm{i}}$-Fab16 dataset (Tsai et al., 2019), and compared the results to the variability analysis performed on the cannabinoid-1 (Punjani and Fleet, 2020), secretin-receptor (Dong et al., 2020) -Gprotein complexes.

The three GPCR-G-protein complexes compared present similarities and differences. Cannabinoid and rhodopsin are class A GPCRs, are bound to $G_{i}$, have the same binder (scFv16 and full Fab16, respectively), and all three complexes are embedded within a LMNG micelle. But they also have important differences. The secretin receptor is a class B GPCR bound to $G_{s}$ and has a nanobody (nb35) bound. While Fab16 binds simultaneously $\mathrm{G} \beta$ and the N-terminal helix of $\mathrm{Gi}$, nb35 binds at a different interface of the $\mathrm{G} \alpha_{s}$ and $\mathrm{G} \beta$ subunit (Rasmussen et al., 2011). The outcome of the variability analysis indicates, however, that these complexes share remarkable similarities in terms of their flexibility, and the movements be categorized within three sets: 1) rotation of the receptor on an axis parallel to the membrane; 2 ) bending of the receptor towards $\mathrm{G} \beta \gamma /$ Fab16 (cannabinoid and rhodopsin) or towards the $\mathrm{N}$ terminal helix of $\mathrm{G}_{\mathrm{s}}$ (secretin) on an axis perpendicular to the membrane; 3) transversal bending of the receptor relative to $G \beta \gamma$. While the overall motions are remarkably similar, a question remains on which component within each dataset contributes for most of the variance observed.

Another important aspect to consider is that these complexes are embedded in a detergent micelle and thus questions remain on whether similar movements, especially those involving the receptors, might be observed in the cell membrane.

The results obtained provide insights into dynamical features of GPCR-G-protein complexes that are not always captured in highresolutions structures. One of these structural features is the AHdomain of $\mathrm{G} \alpha$, a small, helical domain that was visible in an openconformation (displaced from the Ras-domain) in the $B_{2} A R-G_{s}$ crystal structure (Rasmussen et al., 2011), and whose conformational flexibility was characterized by single-particle analysis (Westfield et al., 2011). In
$\mathrm{G} \alpha$ proteins, the nucleotide binds at the interface between the Rasdomain and the AH-domain, and thus stabilizes the AH-domain in its closed conformation. Separation of the AH-domain from the Ras-domain might contribute to increase the probability of nucleotide release (Dror et al., 2015; Sun et al., 2018). Due to the flexible connection between the $\mathrm{AH}$-domain and the Ras-domain, in the absence of nucleotide the $\mathrm{AH}$ domain is expected to fluctuate freely. In MD simulations, the $\mathrm{AH}$ domain was observed to fluctuate from a G $\beta$-bound state to a closedstate (Dror et al., 2015; Sun et al., 2018). In structures of GPCR-Gprotein complexes, the AH-domain is usually not visible either because it was truncated, or because of the mask applied during singleparticle processing. When visible (Kang et al., 2018; Klenk et al., 2020) the position of the AH-domain is in its open conformation bound to G $\beta$, in a similar position to what we observe in the rhodopsin-complex within component one of our analysis (Fig. 1). In our analysis, the movements of the AH-domain propagate through the Ras-domain, and are in synchrony with rotation of rhodopsin on an axis parallel to the membrane.

Another aspect of GPCR-G-protein complexes related to flexibility concerns the interactions at the interface between receptor and G-proteins, especially those interactions mediated by the receptors' intracellular loops (ICL). Structural alignments indicate that ICL2 and ICL3 are often at contact distance to G $\alpha$ (García-Nafría and Tate, 2019; Glukhova et al., 2018). In the structure of the NTR1 receptor- $G_{i}$ complex in the canonical (C) state, ICL2 and ICL3 make a larger number of contacts to $\mathrm{G} \alpha$ as compared to the structure in the non-canonical (NC) state, which is rotated as compared to the C-state (Klenk et al., 2020). In our analysis, the interactions between rhodopsin ICL2 and ICL3 and G $\alpha \beta$ are breaking and forming along the oscillations and rotations observed.

\section{Conclusions}

The characterization of intrinsic protein flexibility and heterogeneity embedded within cryo-EM datasets is becoming a routine analysis performed on the side of the traditional single-particle analysis. Together with data obtained with other methods to study GPCRs (Gusach et al., 2020), this information will contribute to characterize the dynamic nature GPCR-G-protein complex formation, and can be useful to guide the selection of specific binders that limit the degree of freedom between receptor and G-proteins, to facilitate high-resolution structure determination by cryo-EM.

\section{Author contributions}

Jacopo Marino conceived the project with Gebhard F.X. Schertler. Jacopo analyzed data and wrote the manuscript. Gebhard F.X. Schertler helped writing the manuscript.

\section{Funding}

Jacopo Marino acknowledges a Swiss National Science Foundation (SNSF) grant \#19082). Gebhard F.X. Schertler acknowledges SNSF grants \# 310030B_173335 and \# 310030_192760.

\section{Declaration of Competing Interest}

The authors declare that they have no known competing financial interests or personal relationships that could have appeared to influence the work reported in this paper.

\section{Acknowledgments}

Jacopo Marino and Gebhard F.X. Schertler are thankful to the colleagues who contributed to the original elife publication on the rhodopsin-G-protein complex, and to especially Ricardo Adaixo, Filip Pamula, Jonas Mühle, Ching-Ju Tsai, and Xavier Duepi.

\section{Appendix A. Supplementary data}

Supplementary data to this article can be found online at https://doi. 
org/10.1016/j.jsb.2021.107699.

\section{References}

Andén, J., Singer, A., 2018. Structural variability from noisy tomographic projections. SIAM J. Imaging Sci. 11, 1441-1492. https://doi.org/10.1137/17M1153509.

Asarnow, D., Palovcak, E., Cheng, Y., 2019. UCSF pyem v0.5. Zenodo. 10.5281/ zenodo. 3576630

Azevedo, A.W., Doan, T., Moaven, H., Sokal, I., Baameur, F., Vishnivetskiy, S.A., Homan, K.T., Tesmer, J.J.G., Gurevich, V.V., Chen, J., Rieke, F., 2015. C-terminal threonines and serines play distinct roles in the desensitization of rhodopsin, a $\mathrm{G}$ protein-coupled receptor. Elife 4, 1-57. https://doi.org/10.7554/eLife.05981.

Capper, M.J., Wacker, D., 2018. A complex story of receptor signalling G-proteincoupled. Nature 558, 529-530. https://doi.org/10.1038/d41586-018-05503-4.

Costa-Neto, C.M., Parreiras-e-silva, L.T., Bouvier, M., 2016. Pluridimensional view of biased agonism. Mol. Pharmacol. 90, 587-595.

Danev, R., Belousoff, M., Liang, Y., Zhang, X., Wootten, D., Sexton, P.M., 2020. Routine sub-2.5 A cryo-EM structure determination of B-family G protein-coupled receptors.

Dashti, A., Mashayekhi, G., Shekhar, M., Hail, D.B., Salah, S., Schwander, P., Georges, A., Singharoy, A., Frank, J., Ourmazd, A., 2020. Retrieving functional pathways of biomolecules from single-particle snapshots. Nat. Commun. 11 https://doi.org/ 10.1038/s41467-020-18403-X.

Dashti, A., Schwander, P., Langlois, R., Fung, R., Li, W., Hosseinizadeh, A., Liao, H.Y., Pallesen, J., Sharma, G., Stupina, V.A., Simon, A.E., Dinman, J.D., Frank, J., Ourmazd, A., 2014. Trajectories of the ribosome as a Brownian nanomachine. Proc. Natl. Acad. Sci. 111, 17492-17497. https://doi.org/10.1073/pnas.1419276111.

Deupi, X., Kobilka, B.K., 2010. Energy landscapes as a tool to integrate GPCR structure, dynamics, and function. Physiology 25, 293-303. https://doi.org/10.1152/ physiol.00002.2010.

Dong, M., Deganutti, G., Piper, S.J., Liang, Y.L., Khoshouei, M., Belousoff, M.J., Harikumar, K.G., Reynolds, C.A., Glukhova, A., Furness, S.G.B., Christopoulos, A., Danev, R., Wootten, D., Sexton, P.M., Miller, L.J., 2020. Structure and dynamics of the active Gs-coupled human secretin receptor. Nat. Commun. 11, 1-17. https://doi. org/10.1038/s41467-020-17791-4.

Dror, R.O., Mildorf, T.J., Hilger, D., Manglik, A., Borhani, D.W., Arlow, D.H., Philippsen, A., Villanueva, N., Yang, Z., Lerch, M.T., Hubbell, W.L., Kobilka, B.K., Sunahara, R.K., Shaw, D.E., 2015. Structural basis for nucleotide exchange in heterotrimeric G proteins. Science (80-.) 348. https://doi.org/10.1126/science. aaa5264.

Flock, T., Hauser, A.S., Lund, N., Gloriam, D.E., Balaji, S., Babu, M.M., 2017. Selectivity determinants of GPCR-G-protein binding. Nature 545, 317-322. https://doi.org/ 10.1038 /nature2207.

Frank, J., Ourmazd, A., 2016. Continuous changes in structure mapped by manifold embedding of single-particle data in cryo-EM. Methods 100, 61-67. https://doi.org/ 10.1016/j.ymeth.2016.02.007.

García-Nafría, J., Tate, C.G., 2019. Cryo-EM structures of GPCRs coupled to Gs, Gi and Go. Mol. Cell. Endocrinol. 488, 1-13. https://doi.org/10.1016/j.mce.2019.02.006.

Glaeser, R.M., 2018. Proteins, interfaces, and cryo-EM grids. Curr. Opin. Colloid Interface Sci. 34, 1-8. https://doi.org/10.1016/j.cocis.2017.12.009.

Glukhova, A., Draper-Joyce, C.J., Sunahara, R.K., Christopoulos, A., Wootten, D., Sexton, P.M., 2018. Rules of engagement: GPCRs and G proteins. ACS Pharmacol. Transl. Sci. 1, 73-83. https://doi.org/10.1021/acsptsci.8b00026.

Gurevich, V.V., Gurevich, E.V., 2019. GPCR signaling regulation: the role of GRKs and arrestins. Front. Pharmacol. 10, 1-11. https://doi.org/10.3389/fphar.2019.00125.

Gurevich, E.V., Tesmer, J.J.G., Mushegian, A., Gurevich, V.V., 2012. G protein-coupled receptor kinases: more than just kinases and not only for GPCRs. Pharmacol. Ther. 133, 40-69. https://doi.org/10.1016/j.pharmthera.2011.08.001.

Gusach, A., Maslov, I., Luginina, A., Borshchevskiy, V., Mishin, A., Cherezov, V., 2020. Beyond structure: emerging approaches to study GPCR dynamics. Curr. Opin. Struct. Biol. 63, 18-25. https://doi.org/10.1016/j.sbi.2020.03.004.

Haie-Meder, C., 1998. EM algorithms for PCS and SPCA. Adv. Neural Inf. Process. Syst. 626-632 https://doi.org/10.1016/s0167-8140(11)70666-1.

Hauser, A.S., Attwood, M.M., Rask-Andersen, M., Schiöth, H.B., Gloriam, D.E., 2017. Trends in GPCR drug discovery: new agents, targets and indications. Nat. Rev. Drug Discov. 16, 829-842. https://doi.org/10.1038/nrd.2017.178.

Huang, W., Masureel, M., Qu, Q., Janetzko, J., Inoue, A., Skiniotis, G., Kobilka, B.K., 2020. Structure of the neurotensin receptor 1 in complex with $\beta$-arrestin 1 . Nature 579. https://doi.org/10.1038/s41586-020-1953-1.

Jin, Q., Sorzano, C.O.S., de la Rosa-Trevín, J.M., Bilbao-Castro, J.R., Núñez-Ramírez, R., Llorca, O., Tama, F., Jonić, S., 2014. Iterative elastic 3D-to-2D alignment method using normal modes for studying structural dynamics of large macromolecular complexes. Structure 22, 496-506. https://doi.org/10.1016/j.str.2014.01.004.

Joseph P. Neol, H.E.H. \& P.B.S., 1993. The 2.2 A crystal structure of transducin-a complexed with GTPyS. Nature 366, 654-663.

Kang, Y., Kuybeda, O., De Waal, P.W., Mukherjee, S., Van Eps, N., Dutka, P., Zhou, X.E., Bartesaghi, A., Erramilli, S., Morizumi, T., Gu, X., Yin, Y., Liu, P., Jiang, Y., Meng, X., Zhao, G., Melcher, K., Ernst, O.P., Kossiakoff, A.A., Subramaniam, S., Xu, H.E., 2018. Cryo-EM structure of human rhodopsin bound to an inhibitory G protein. Nature 558, 553-558. https://doi.org/10.1038/s41586-018-0215-y.

Klenk, C., Merklinger, L., Morstein, L., Hagn, F., Plückthun, A., Zhang, M., Gui, M., Wang, Z.-F., Gorgulla, C., Yu, J.J., Wu, H., Sun, Z., Klenk, C., Merklinger, L. Morstein, L., Hagn, F., Plückthun, A., Brown, A., Nasr, M.L., Wagner, G., 2020. CryoEM structure of an activated GPCR-G protein complex in lipid nanodiscs. bioRxiv. https://doi.org/10.1101/2020.06.11.145912.
Kontziampasis, D., Klebl, D.P., Iadanza, M.G., Scarff, C.A., Kopf, F., Sobott, F., Monteiro, D.C.F., Trebbin, M., Muench, S.P., White, H.D., 2019. A cryo-EM grid preparation device for time-resolved structural studies. IUCrJ 6, 1024-1031. https:// doi.org/10.1107/S2052252519011345.

Krishna Kumar, K., Shalev-Benami, M., Robertson, M.J., Hu, H., Banister, S.D., Hollingsworth, S.A., Latorraca, N.R., Kato, H.E., Hilger, D., Maeda, S., Weis, W.I., Farrens, D.L., Dror, R.O., Malhotra, S.V., Kobilka, B.K., Skiniotis, G., 2019. Structure of a signaling cannabinoid receptor 1-G protein complex. Cell 176, 448-458.e12. https://doi.org/10.1016/j.cell.2018.11.040.

Latorraca, N.R., Venkatakrishnan, A.J., Dror, R.O., 2017. GPCR dynamics: structures in motion. Chem. Rev. 117, 139-155. https://doi.org/10.1021/acs.chemrev.6b00177.

Lee, Y., Warne, T., Nehmé, R., Pandey, S., Dwivedi-agnihotri, H., Chaturvedi, M., Edwards, P.C., García-nafría, J., Leslie, A.G.W., Shukla, A.K., Tate, C.G., 2020. Molecular basis of $\beta$-arrestin coupling to. Nature 583. https://doi.org/10.1038/ s41586-020-2419-1.

Liang, Y.L., Belousoff, M.J., Fletcher, M.M., Zhang, X., Khoshouei, M., Deganutti, G., Koole, C., Furness, S.G.B., Miller, L.J., Hay, D.L., Christopoulos, A., Reynolds, C.A., Danev, R., Wootten, D., Sexton, P.M., 2020. Structure and dynamics of adrenomedullin receptors AM1 and AM2 reveal key mechanisms in the control of receptor phenotype by receptor activity-modifying proteins. ACS Pharmacol. Transl. Sci. 3, 263-284. https://doi.org/10.1021/acsptsci.9b00080.

Lyumkis, D., 2019. Challenges and opportunities in cryo-EM single-particle analysis. J. Biol. Chem. 294, 5181-5197. https://doi.org/10.1074/jbc.REV118.005602.

Maeda, S., Qu, Q., Robertson, M.J., Skiniotis, G., Kobilka, B.K., 2019. Structures of the M1 and M2 muscarinic acetylcholine receptor/G-protein complexes. Science (80-.) 364, 552-557. https://doi.org/10.1126/science.aaw5188.

Nass Kovacs, G., Colletier, J.P., Grünbein, M.L., Yang, Y., Stensitzki, T., Batyuk, A., Carbajo, S., Doak, R.B., Ehrenberg, D., Foucar, L., Gasper, R., Gorel, A., Hilpert, M., Kloos, M., Koglin, J.E., Reinstein, J., Roome, C.M., Schlesinger, R., Seaberg, M., Shoeman, R.L., Stricker, M., Boutet, S., Haacke, S., Heberle, J., Heyne, K., Domratcheva, T., Barends, T.R.M., Schlichting, I., 2019. Three-dimensional view of ultrafast dynamics in photoexcited bacteriorhodopsin. Nat. Commun. 10 https://doi. org/10.1038/s41467-019-10758-0.

Nešić, D., Zhang, Y., Spasic, A., Li, J., Provasi, D., Filizola, M., Walz, T., Coller, B.S., 2020. Cryo-electron microscopy structure of the $\alpha$ iIb $\beta 3$-abciximab complex. Arterioscler. Thromb. Vasc. Biol. 11, 624-637. https://doi.org/10.1161/ ATVBAHA.119.313671.

Nguyen, A.H., Thomsen, A.R.B., Iii, T.J.C., Huang, R., Huang, L., Marcink, T., Clarke, O. B., Heissel, S., Masoudi, A., Ben-hail, D., Samaan, F., 2019. Structure of an endosomal signaling GPCR-G protein- $\beta$-arrestin megacomplex. Nat. Struct. Mol. Biol. 26 https://doi.org/10.1038/s41594-019-0330-y.

Nogly, P., Weinert, T., James, D., Carbajo, S., Ozerov, D., Furrer, A., Gashi, D., Borin, V., Skopintsev, P., Jaeger, K., Nass, K., Båth, P., Bosman, R., Koglin, J., Seaberg, M., Lane, T., Kekilli, D., Brünle, S., Tanaka, T., Wu, W., Milne, C., White, T., Barty, A., Weierstall, U., Panneels, V., Nango, E., Iwata, S., Hunter, M., Schapiro, I., Schertler, G., Neutze, R., Standfuss, J., 2018. Retinal isomerization in bacteriorhodopsin captured by a femtosecond x-ray laser. Science 80-, 361. https:// doi.org/10.1126/science.aat0094.

Penczek, P.A., Kimmel, M., Spahn, C.M.T., 2011. Identifying conformational states of macromolecules by Eigen-analysis of resampled cryo-EM images. Structure 19, 1582-1590. https://doi.org/10.1016/j.str.2011.10.003.

Pettersen, E.F., Goddard, T.D., Huang, C.C., Couch, G.S., Greenblatt, D.M., Meng, E.C., Ferrin, T.E., 2004. UCSF Chimera-a visualization system for exploratory research and analysis. J. Comput. Chem. 25, 1605-1612. https://doi.org/10.1002/jcc.20084.

Punjani, A., Fleet, D.J., 2020. 3D variability analysis: directly resolving continuous flexibility and discrete heterogeneity from single particle cryo-EM images. bioRxiv. https://doi.org/10.1101/2020.04.08.032466.

Punjani, A., Rubinstein, J.L., Fleet, D.J., Brubaker, M.A., 2017. cryoSPARC: algorithms for rapid unsupervised cryo-EM structure determination. Nat. Methods 14, 290-296. https://doi.org/10.1038/nmeth.4169.

Qu, X., Wang, D., Wu, B., 2019. In: Progress in GPCR structure determination, GPCRs: Structure, Function, and Drug Discovery. Elsevier Inc. https://doi.org/10.1016/ B978-0-12-816228-6.00001-5

Rajagopal, S., Rajagopal, K., Lefkowitz, R.J., 2010. Teaching old receptors new tricks: Biasing seven-transmembrane receptors. Nat. Rev. Drug Discov. 9, 373-386. https:// doi.org/10.1038/nrd3024.

Ramírez, A.S., Kowal, J., Locher, K.P., 2019. Cryo-electron microscopy structures of human oligosaccharyltransferase complexes OST-A and OST-B. Science (80-.) 366, 1372-1375. https://doi.org/10.1126/science.aaz3505.

Rasmussen, S.G.F., Devree, B.T., Zou, Y., Kruse, A.C., Chung, K.Y., Kobilka, T.S., Thian, F. S., Chae, P.S., Pardon, E., Calinski, D., Mathiesen, J.M., Shah, S.T.A., Lyons, J.A., Caffrey, M., Gellman, S.H., Steyaert, J., Skiniotis, G., Weis, W.I., Sunahara, R.K., Kobilka, B.K., 2011. Crystal structure of the $\beta 2$ adrenergic receptor-Gs protein complex. Nature 477, 549-557. https://doi.org/10.1038/nature10361.

Rogala, K.B., Gu, X., Kedir, J.F., Abu-Remaileh, M., Bianchi, L.F., Bottino, A.M.S., Dueholm, R., Niehaus, A., Overwijn, D., Priso Fils, A.C., Zhou, S.X., Leary, D Laqtom, N.N., Brignole, E.J., Sabatini, D.M., 2019. Structural basis for the docking of mTORC1 on the lysosomal surface. Science (80-.) 366, 468-475. https://doi.org/ 10.1126/science.aay0166.

Scheres, S.H.W., 2016. Processing of Structurally Heterogeneous Cryo-EM Data in RELION. In: Methods in Enzymology, first ed. Elsevier Inc. https://doi.org/10.1016/ bs.mie.2016.04.012.

Sexton, P.M., 2018. Mechanisms of signalling and biased agonism in G protein-coupled receptors. Nat. Rev. Mol. Cell Biol. 19 https://doi.org/10.1038/s41580-018-0049-3. Sexton, P.M., Christopoulos, A., 2018. To bind or not to bind: unravelling GPCR polypharmacology. Cell 172, 636-638. https://doi.org/10.1016/j.cell.2018.01.018. 
Stanishneva-Konovalova, T.B., Semenyuk, P.I., Kurochkina, L.P., Pichkur, E.B., Vasilyev, A.L., Kovalchuk, M.V., Kirpichnikov, M.P., Sokolova, O.S., 2020. Cryo-EM reveals an asymmetry in a novel single-ring viral chaperonin. J. Struct. Biol. 209, 107439 https://doi.org/10.1016/j.jsb.2019.107439.

Sun, X., Singh, S., Blumer, K.J., Bowman, G.R., 2018. Simulation of spontaneous G protein activation reveals a new intermediate driving GDP unbinding. Elife 7, 1-24. https://doi.org/10.7554/eLife.38465.

Sungkaworn, T., Jobin, M., Burnecki, K., Weron, A., Lohse, M.J., Calebiro, D., 2017. Single-molecule imaging reveals receptor-G protein interactions at cell surface hot spots. Nat. Publ. Gr. 550, 543-547. https://doi.org/10.1038/nature24264.

Tagare, H.D., Kucukelbir, A., Sigworth, F.J., Wang, H., Rao, M., 2015. Directly reconstructing principal components of heterogeneous particles from cryo-EM images. J. Struct. Biol. 191, 245-262. https://doi.org/10.1016/j.jsb.2015.05.007.

Thal, D.M., Vuckovic, Z., Draper-joyce, C.J., Liang, Y., Glukhova, A., Christopoulos, A., Sexton, P.M., 2018. Recent advances in the determination of $G$ protein-coupled receptor structures. Curr. Opin. Struct. Biol. 51, 28-34. https://doi.org/10.1016/j. sbi.2018.03.002.

Tsai, C.-J., Marino, J., Adaixo, R., Pamula, F., Muehle, J., Maeda, S., Flock, T., Taylor, N. M.I., Mohammed, I., Matile, H., Dawson, R.J.P., Deupi, X., Stahlberg, H.,

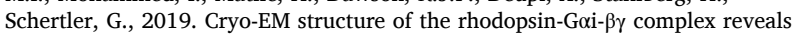
binding of the rhodopsin C-terminal tail to the G $\beta$ subunit. Elife 8, e46041. https:// doi.org/10.7554/eLife.46041.

Unwin, N., Fujiyoshi, Y., 2012. Gating movement of acetylcholine receptor caught by plunge-freezing. J. Mol. Biol. 422, 617-634. https://doi.org/10.1016/j. jmb.2012.07.010.
Violin, J.D., Crombie, A.L., Soergel, D.G., Lark, M.W., 2014. Biased ligands at G-proteincoupled receptors: Promise and progress. Trends Pharmacol. Sci. 35, 308-316. https://doi.org/10.1016/j.tips.2014.04.007.

Vögler, O., Barceló, J.M., Ribas, C., Escribá, P.V., 2008. Membrane interactions of G proteins and other related proteins. Biochim. Biophys. Acta, Gene Struct. Expression 1778, 1640-1652. https://doi.org/10.1016/j.bbamem.2008.03.008.

Wacker, D., Stevens, R.C., Roth, B.L., 2017. Review how ligands illuminate GPCR molecular pharmacology. Cell 170, 414-427. https://doi.org/10.1016/j. cell.2017.07.009.

Westfield, G.H., Rasmussen, S.G.F., Su, M., Dutta, S., Devree, B.T., Chung, K.Y., Calinski, D., Velez-Ruiz, G., Oleskie, A.N., Pardon, E., Chae, P.S., Liu, T., Li, S., Woods, V.L., Steyaert, J., Kobilka, B.K., Sunahara, R.K., Skiniotis, G., 2011. Structural flexibility of the Gos $\alpha$-helical domain in the $\beta 2$-adrenoceptor Gs complex. Proc. Natl. Acad. Sci. 108 https://doi.org/10.1073/pnas.1113645108.

Wrapp, D., Wang, N., Corbett, K.S., Goldsmith, J.A., Hsieh, C.L., Abiona, O., Graham, B. S., McLellan, J.S., 2020. Cryo-EM structure of the 2019-nCoV spike in the prefusion conformation. Science (80-.) 367, 1260-1263. https://doi.org/10.1126/science. aax0902.

Zhang, C., Cantara, W., Jeon, Y., Musier-Forsyth, K., Grigorieff, N., Lyumkis, D., 2019. Analysis of discrete local variability and structural covariance in macromolecular assemblies using Cryo-EM and focused classification. Ultramicroscopy 203, 170-180. https://doi.org/10.1016/j.ultramic.2018.11.016. 